\title{
Plenary lecture
}

\section{Wednesday, May 9, 2012 \\ Opening Plenary Lecture}

39 invited speaker

FUTURE TRENDS IN CLINICAL RESEARCH AND MANAGEMENT A. BASDEVANT ${ }^{1}$

${ }^{1}$ INSTITUTE OF CARDIO-METABOLISM AND NUTRITION, ICAN,PARIS-6 UNIVERSITY, HÔPITAL DE LA PITIÉ SALPÊTRIĖRE-PARIS, NUTRITION, Paris, France

Abstract Text: The impact of obesity and of its related complications on public health calls for an integrated research approach spaning from basic to social sciences and from clinical research to care organisation innovation. The objective is to develop integrated strategies to improve prevention and promote an "obesity medicine" based upon the identification of preclinical events, predictors of responses to treatment and innovative diagnostic tools and therapy. Medical interventions should intervene earlier in the natural cycle of the disease, before irreversible damages develop. There are still unmet needs in the physiopathological knowledge, the diagnosis and treatment. New actors came into the scene as the role of gut microbiota, pollutants or other environmental factors. The challenge is to manage obesity over the entire life cycle, to identify individual's susceptibility, to prevent, early diagnose and pre-empt complications with a protection of body tissue damage as long as possible. The resulting clinical goal is a treatment adapted to the stage of the diseases and individuals' characteristics (genetic, state of tissue damage, lifestyle etc). This requires a comprehensive patient phenotyping using modern technologies taking in account the dynamics and multifactorial nature of obesity and related complications. We have to turn from "one treatment fits for all" to an individually tailored approach. Conflict of Interest: No conflict of interest

Funding:

\section{Thursday, May 10, 2012}

\section{T2:PL - Track 2 Plenary Lecture}

41 invited speaker

EPIDEMIOLOGIC EVIDENCE ON THE LONG TERM HARMS AND BENEFITS OF BARIATRIC SURGERY

E. Näslund $^{1}$

${ }^{1}$ KAROLINSKA INSTITUTET, Department of clinical sciences, Danderyd Hospital, Stockholm, Sweden

Abstract Text: The number of persons undergoing bariatric surgery increases on a yearly bases. It was estimated that 150000 persons underwent bariatric surgery in the world in 2003 and this number was doubled in 2008. It is unlikely that there will be any large randomized trials comparing bariatric surgery to conventional treatment. In light of this large population based studies with a non-treated control group is the second best thing. This talk will highlight some population-based studies, strength and weaknesses of population-based studies as well as published and unpublished results from large population-based studies. Both benefits and harms of bariatric surgery will be discussed. Based on these studies (and others), Sweden has recently amended the nationwide guidelines for bariatric surgery to $\mathrm{BMI}>35 \mathrm{~kg} / \mathrm{m}^{2}$ without co-morbidity. The reasoning behind this will be discussed.

Conflict of Interest: None

Funding: 


\section{Friday, May 11, 2012}

\section{T3:PL - Track 3 Plenary Lecture}

42 invited speake

\section{GLP1: FROM BENCH TO PATIENTS}

J. J. Holst $^{1}$

1 MD, NNF Center for Basic Metabolic Research, Dept BIOMEDICAL SCIENCES, UNIVERSITY OF Copenhagen, the PANUM INSTITUTE., Copenhagen, Denmark

Abstract Text: Glucagon-like peptide-1 (GLP-1) was identified as an insulin-releasing substance from the gut, but turned out to have many more actions, including inhibition of glucagon secretion, inhibition of gastrointestinal secretion and motility, and inhibition of appetite/food intake. It exerts its effect via activation of the single GLP-1 receptor, expressed in the pancreatic islets, but also in the central nervous system and on sensory vagal afferent neurons. Recent studies involving the GLP-1 receptor antagonist exendin 9-39 have shown that all the mentioned effects are likely to be regulated by endogenous GLP-1. Thus, GLP-1 must be counted as one the appetite-regulating hormones, and its role in the pathogenesis of obesity and diabetes is being investigated, as well as the mechanisms whereby it inhibits food intake. But does GLP-1 also have a role in the treatment of obesity? Numerous clinical studies in patients with type 2 diabetes (T2DM) have shown that the available GLP-1 receptor agonists (GLP-1 RA: exenatide, bid; exenatide once weekly; and liraglutide) not only improve glycemic levels but also cause a moderate weight loss. Recent studies with both exenatide and liraglutide have demonstrated robust weight losses also in individuals with obesity without T2DM. Interestingly, glucose tolerance, if impaired, was normalized, suggesting that treatment with GLP-1 may prevent development of glucose intolerance/T2DM. The weight loss induced by GLP-1 RA is durable for at least 2 years, and GLP-1 RAs may help stabilize a weight loss induced by calorie restriction. Conflict of Interest:

Funding:

\section{T4:PL - Track 4 Plenary Lecture}

43 invited speaker

LEPTIN AND THE HOMEOSTATIC SYSTEM REGULATING BODY WEIGHT

J. Friedman

1 HHMI/Rockefeller University, Molecular Genetics, New York, USA

Abstract Text: The discovery of leptin has led to the elucidation of a robust physiologic system that maintains fat stores at a relatively constant level. Leptin is a peptide hormone secreted by adipose tissue in proportion to its mass. This hormone circulates in blood and acts on the hypothalamus to regulate food intake and energy expenditure. When fat mass falls, plasma leptin levels fall stimulating appetite and suppressing energy expenditure until fat mass is restored. When fat mass increases, leptin levels increase, suppressing appetite until weight is lost. By such a mechanism total energy stores are stably maintained within a relatively narrow range. Recessive mutations in the leptin gene are associated with massive obesity in mice and some humans. Treatment with recombinant leptin markedly reduces food intake and body weight. The low leptin levels in patients with leptin mutations are also associated with multiple abnormalities including infertility, diabetes and immune abnormalities all of which are corrected by leptin treatment. These findings have established important links between energy stores and many other physiologic systems and led to the use of leptin as a treatment for an increasing number of other human conditions including a subset of obesity, some forms of diabetes including lipodystrophy and hypothalamic amennorhea, the cessation of menstruation seen in extremely thin women. Identification of a physiologic system that controls energy balance establishes a biologic basis for obesity and further establishes links between leptin and numerous other physiologic responses. Recent studies have explored the relationship between leptin and the reward value of food. In addition, new methods for identifying neurons activated by leptin and other stimuli have been developed.

Conflict of Interest:

Funding:

\section{Saturday, May 12, 2012}

\section{General Plenary Lecture}

44 invited speaker

STANDING UP FOR SEDENTARY BEHAVIOUR IN OBESITY RESEARCH

P. Katzmarzyk ${ }^{1}$

$\overline{1}$ Pennington Biomedical Research Center, Baton Rouge, USA

Abstract Text: The emergence of obesity as a major public health issue has prompted efforts to understand the contributions of both energy intake and expenditure to the regulation of body weight. On the energy expenditure side of the equation, an emphasis has been placed on understanding the role of moderate-to-vigorous physical activity levels in the prevention and management of obesity and related disorders. However, there is emerging evidence for a significant role for sedentary behaviors, which predominantly involve extended periods of sitting, and the development of obesity, type 2 diabetes, cardiovascular disease, as well as premature mortality. Epidemiological evidence indicates that sedentary behaviour may be a distinct, independent risk factor for chronic disease, beyond the protective effects associated with moderate-to-vigorous physical activity. Further, extended periods of sitting result in increased health risks among normal weight, overweight and obese adults, suggesting that the effects may be independent of body weight status per se. Further research is required to better delineate the mechanisms linking sedentary behavior to health, and the extent to which it is an independent risk factor, separate from the effects of obesity.

Conflict of Interest: No conflict of interest.

Funding:

\section{T5:PL - Track 5 Plenary Lecture: Debate}

45 invited speaker

PHARMACOLOGIC MANAGEMENT OF OBESITY: EFFICACY VERSUS SAFETY

A. Astrup ${ }^{1}$

${ }^{1}$ University of Copenhagen, Faculty of SCIENCE, Department of Human Nutrition, Frederiksberg, Denmark

Abstract Text: The development of drugs for the management of obesity has been hampered by too much focus on efficacy and too little attention paid to safety. This has led to a series of withdrawals of drugs from the market after serious adverse effects became apparent. Rimonabant was withdrawn in Europe after the discovery of an increased risk of depression, anxiety and suicidal ideation. These serious adverse events were class effects and the development of several other compounds of the same class was terminated. One might wonder why it was independent scientists who discovered the severity of these adverse effects. In January 2010 the European Medicine Agency's Committee for Medicinal Products for Human Use (EMA) recommended the suspension of sibutramine, a weight loss agent known to produce a slight increase in blood pressure and heart rate, and the drug was withdrawn from the European market. The reason was results of the SCOUT study, which was conducted at the request of EMA, on the effect of sibutramine on hard end-points in 10,000 overweight and obese patients with cardiovascular disease and type 2 diabetes over 6 years. The analysis found an $18 \%$ increased risk of serious, non-fatal cardiovascular events, such as stroke or heart attack, with sibutramine compared to placebo. However, in subsequent analyses it was found that a modest weight loss over both short-term and longer-term (6-12 months) periods was associated with reduction in cardiovascular mortality for the following 4-5 years even in those with pre-existing cardiovascular disease. It is clear that the increased cardiovascular risk was due to those patients who did not lose weight. How many doctors would continue to treat obese patients with weight loss drugs if they don't lose weight? This example emphasizes the need to bring in obesity experts as advisors to regulators. There are several examples where the guidelines for clinical investigations of weight loss drugs demonstrate a lack of understanding of the clinical pharmacology of obesity management. EMEA: "Demonstration of a significant degree of weight loss of at least $10 \%$ of baseline weight, which is also statistically significantly greater than that associated with placebo, is considered to be a valid efficacy criterion in clinical trials evaluating new anti-obesity drugs." This can 
quite easily be achieved by a dietary fibre preparation, as the weight loss from baseline is mainly determined by the hypocaloric diet program and very little efficacy of the drug is needed to get a greater weight loss than placebo.Finally, BMI is an inadequate measure of degree of obesity and comorbidity risk, and regulators should accept The Edmonton Obesity Staging System for classifying obesity based on clinical parameters as a guide to management when translating the outcome of obesity trials into clinical practice.

Conflict of Interest: Arne Astrup is consultant/member of advisory boards for Pathway Genomics Corporation, USA; 7TM Pharma, DK; Arena Pharmaceuticals Inc., USA; Doyen Medical Inc., USA; NeuroSearch, DK; Novo Nordisk, DK; Orexigen Therapeutics Inc., USA; Rhytm Pharmaceuticals, USA; S-Biotek, DK; Twinlab, USA; Vivus Inc., USA

Funding: His research group is currently conducting a clinical trial funded by Novo Nordic A/S, Denmark, and has previously conducted trials for other pharmaceutical companies. 